\title{
Improving Aerosol Drug Delivery During Invasive Mechanical Ventilation With Redesigned Components
}

\author{
P Worth Longest PhD, Mandana Azimi PharmD, Laleh Golshahi PhD, and Michael Hindle PhD
}

\begin{abstract}
INTRODUCTION: Patients receiving invasive mechanical ventilation with an endotracheal tube (ETT) can often benefit from pharmaceutical aerosols; however, drug delivery through the ventilator circuit is known to be very inefficient. The objective of this study was to improve the delivery of aerosol through an invasive mechanical ventilation system by redesigning circuit components using a streamlining approach. METHODS: Redesigned components were the T-connector interface between the nebulizer and ventilator line and the Y-connector leading to the ETT. The streamlining approach seeks to minimize aerosol deposition and loss by eliminating sharp changes in flow direction and tubing diameter that lead to flow disruption. Both in vitro experiments and computational fluid dynamic (CFD) simulations were applied to analyze deposition and emitted dose of drug for multiple droplet size distributions, flows, and ETT sizes used in adults. RESULTS: The experimental results demonstrated that the streamlined components improved delivery through the circuit by factors ranging from 1.3 to 1.5 compared with a commercial system for adult ETT sizes of 8 and $9 \mathrm{~mm}$. The overall delivery efficiency was based on the bimodal aspect of the aerosol distributions and could not be predicted by median diameter alone. CFD results indicated a 20-fold decrease in turbulence in the junction region for the streamlined $\mathrm{Y}$ resulting in a maximum 9-fold decrease in droplet deposition. The relative effectiveness of the streamlined designs was found to increase with increasing particle size and increasing flow, with a maximum improvement in emitted dose of 1.9-fold. CONCLUSIONS: Streamlined components can significantly improve the delivery of pharmaceutical aerosols during mechanical ventilation based on an analysis of multiple aerosol generation devices, ETT sizes, and flows. Key words: pharmaceutical aerosols; respiratory drug delivery; mesh nebulizer; mechanical ventilation; T-connector; $Y$-connector; streamlined designs; endotracheal tube; improved aerosol delivery. [Respir Care 2014;59(5):686-698. (C) 2014 Daedalus Enterprises]
\end{abstract}

\section{Introduction}

Respiratory drug delivery during mechanical ventilation with an endotracheal tube (ETT; invasive mechanical ven-

\footnotetext{
Dr Longest is affiliated with the Department of Mechanical and Nuclear Engineering, and Drs Longest, Azimi, Golshahi, and Hindle are affiliated with the Department of Pharmaceutics, Virginia Commonwealth University, Richmond, VA.

Dr Golshahi presented a version of this work at the 19th International Congress of the International Society for Aerosols in Medicine, held April 2013 in Chapel Hill, North Carolina.
}

This study was supported by Award R01 HL107333 from the National Heart, Lung, and Blood Institute.

All authors are employees of Virginia Commonwealth University. Virginia Commonwealth University is seeking patent applications with re- tilation) is known to be inefficient based on in vitro and in vivo studies. ${ }^{1-8}$ Dhand $^{1}$ reviews factors that can influence and limit aerosol delivery to the lungs during mechanical ventilation including ventilator settings, circuit design, and aerosol device selection. In general, delivery

\footnotetext{
spect to the technology described in this article, which, if licensed, may result in a financial interest to the authors.

The content of this article is solely the responsibility of the authors and does not necessarily represent the official views of the National Heart, Lung, and Blood Institute or the National Institutes of Health.

Correspondence: P Worth Longest PhD, Virginia Commonwealth University, 401 West Main Street, P.O. Box 843015, Richmond, VA 232843015. E-mail: pwlongest@vcu.edu.
}

DOI: $10.4187 /$ respcare. 02782 
efficiency for most common administration techniques during mechanical ventilation range from 6 to $10 \%$ in vivo; however, even at these low delivery efficiencies, some aerosolized medications are shown to be clinically effective at improving lung function. ${ }^{2}$ Dhand $^{3}$ reviews newer delivery devices including ultrasonic and vibrating mesh nebulizers for use during mechanical ventilation, with actuation timed to coincide with inhalation. For all delivery devices, differences between in vitro predictions and measured in vivo lung deposition during mechanical ventilation are observed, with the inclusion of humidity reducing lung delivery efficiency by $\sim 40 \% .^{3}$ Ari and Fink ${ }^{4}$ provide an updated description of best practices for aerosol delivery during mechanical ventilation, including advances in the timing of activation and device selection, as well as a review of drug efficacy.

Considering specific devices, lung delivery efficiency values for pressurized metered-dose inhalers and nebulizers are typically $<10 \%$ during all forms of mechanical ventilation. $^{2-4,9,10}$ Vibrating mesh nebulizers have significantly increased aerosol delivery efficiencies to values in the range of $10-25 \%$ based on in vitro studies of invasive ventilation. ${ }^{11,12}$ Inhalation triggering of a vibrating mesh nebulizer coupled with aerosol delivery during a portion of the inspiration flow further increased delivery efficiency to values as high as $60 \% .^{3}$ However, one disadvantage of breath-activated mesh nebulizers is the relative cost and complexity of the system compared with other aerosol delivery technologies.

Previous studies ${ }^{3,9}$ have observed high aerosol losses during mechanical ventilation in the aerosol source adapter, connectors, and ETT. Based on these high depositional losses, it can be concluded that current commercial ventilator circuits are not designed for the efficient delivery of aerosols. A practical and cost-effective method to improve aerosol delivery efficiency during mechanical ventilation is to redesign the ventilator circuit components responsible for high aerosol deposition in ways that do not have an adverse effect on gas delivery. In a United States patent, Ivri and Fink ${ }^{13}$ suggest that low angles at ventilator circuit transition points $\left(<15^{\circ}\right)$ can be used to improve aerosol delivery during mechanical ventilation. Alternatively, a previous study by Longest et al ${ }^{14}$ proposes a streamlining approach to ventilator circuit components that eliminates sudden expansions and contractions in the flow path and applies a radius of curvature to changes in flow direction. This approach allows for large angle changes in the flow pathway that are often necessary for connections between the ventilator and patient. The streamlined (SL) component approach results in significantly more uniform flow with less flow separation, detachment, recirculation, vorticity, turbulence, and decreased aerosol deposition by dispersion and inertial impaction. The study by Longest et al ${ }^{14}$ demonstrated that the streamlining approach could reduce

\section{QUICK LOOK}

\section{Current knowledge}

A number of therapeutic aerosolized medications are delivered to mechanically ventilated patients with varying degrees of success. The endotracheal tube (ETT) and ventilator circuit act as a filter that reduces drug delivery to the lungs.

\section{What this paper contributes to our knowledge}

Streamlining of circuit components minimizes flow disruption by avoiding sudden expansions and contractions in the flow stream and eliminating sharp changes in flow direction. For a range of potential polydisperse size distributions and ETTs, a 1.1- to 1.5-fold increase in emitted dose was observed.

deposition within individual components of a high-flow oxygen therapy system by factors as large as 4 times. Overall, delivery efficiency through the high-flow oxygen system was improved by $40 \%$ for conventional sized aerosols based on in vitro experiments by simply using the SL components. However, previous studies have not considered redesigning ventilator circuit components using a streamlining approach to improve the delivery of aerosols during invasive mechanical ventilation. It is questioned whether upstream improvements in aerosol delivery can be maintained through an ETT, which is known to be a region of high particle loss that is not likely to be modified. Furthermore, different ETT sizes will result in different delivery efficiencies to the lung for both commercial and SL ventilator components.

The objective of this study is to evaluate the effects of SL circuit components on the delivery of aerosols during invasive mechanical ventilation conditions in adults. Components considered were the T-connector interface between the nebulizer and ventilator line and the Y-connector leading to the ETT. Multiple ETT sizes used in adult ventilation setups were evaluated along with different representative steady-state air-flows. In vitro experiments were used to evaluate the performance of two mesh nebulizers across a range of ETT sizes for commercial and SL components. Computational fluid dynamic (CFD) simulations were used to explore differences in flow fields created by the SL components compared with the commercial counterparts. In vitro experiments with realistic polydisperse aerosols were used to validate CFD predictions in terms of drug mass deposition. The CFD model was then used to further explore the effectiveness of the SL components for different flows with a series of hypothetical monodisperse aerosols and a monomodal polydisperse aerosol. Results are presented in terms of deposition fractions (DFs) within 


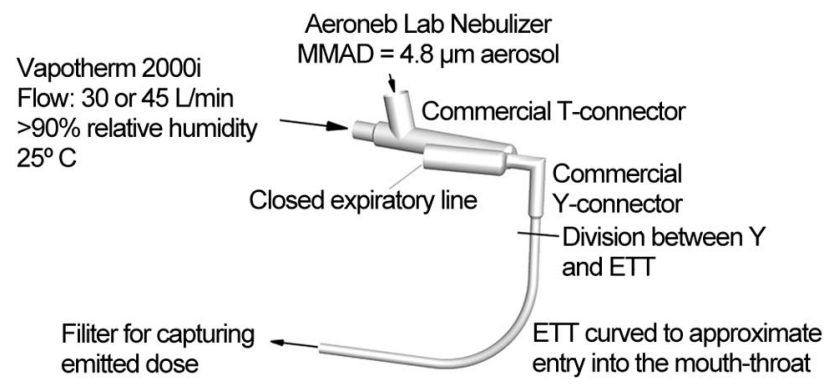

Fig. 1. Experimental setup illustrating the commercial T-connector coupled with a commercial Y-connector and $8 \mathrm{~mm}$ endotracheal tube (ETT). Curvature of the ETT simulates passing through the mouth and throat during invasive mechanical ventilation. MMAD = mass median aerodynamic diameter.

individual system components, emitted dose (ED), and improvements in DF and ED associated with the SL designs. While steady-state air-flow conditions were used in the current study, it is expected that the improvement factors associated with the SL components can be applied to predict the relative performance achieved when these designs are applied to other more realistic conditions, such as with cyclic flow and in vivo aerosol delivery.

\section{Methods}

\section{Ventilation System Components}

Aerosols were generated using two commercial vibrating mesh nebulizers. This form of aerosol production was selected based on previously observed improved performance in mechanical ventilation systems as well as rapid nebulization, low residual volume, and minimum temperature change in the nebulizer solution. ${ }^{11,12}$ The two mesh nebulizers considered were the Aeroneb Lab (Aerogen Limited, Galway, Ireland) and the eFlow Rapid (Pari Respiratory Equipment, Midlothian, Virginia). The Aeroneb Lab device is known to produce a smaller aerosol than the other available Aeroneb mesh devices and was selected to maximize delivery performance in both the CM and SL delivery systems. The eFlow Rapid is not currently intended for aerosol delivery during mechanical ventilation, but was selected to provide a mesh-generated aerosol from a second commercial source. Based on reported variability in aerosol sizes produced by nebulizer devices of the same type and model, ${ }^{4}$ the polydisperse size distribution of both aerosol generation devices was determined in this study using cascade impaction.

An overview of the ventilation circuit considered in this study is provided in Figure 1. In the experiments, temperature-controlled and humidified air was supplied to the circuit. The Aeroneb nebulizer was connected to the $22-\mathrm{mm}$ ventilator line using the commercially available adult
(Aerogen Limited) or the newly designed SL T-connector. Based on manufacturer recommendations for the Aeroneb mesh nebulizers, the T-connector was positioned immediately next to the Y-connector (see Fig. 1). The eFlow device did not have a commercial device for connecting to ventilator tubing. Therefore, the $2 \mathrm{~cm}$ circular outlet of the device was attached directly to the Y-connector as a second source of aerosol generation. The Y-connector was either a commercial version (Teleflex Medical Adult YConnector, Hudson RCI, Research Triangle Park, North Carolina) or a new SL design, and led to ETTs with internal diameters of 7,8 , or $9 \mathrm{~mm}$, which provides a range of tube sizes commonly implemented in adults. To represent the passage of the ETT through the oropharyngeal region, the tubes were curved through a $90^{\circ}$ bend with radii of curvatures equal to 5,8 , and $10 \mathrm{~cm}$ for the 7-, 8-, and $9-\mathrm{mm}$ tubes, respectively. These radii of curvature values are based on small, medium, and large oropharyngeal geometries previously reported by Delvadia et al..$^{15}$ It is not clear whether previous in vitro studies reporting ETT deposition implemented a full $90^{\circ}$ bend of the tube and what radii of curvature values were implemented. The ETTs implemented in this study were manufactured by Hudson RCI/Teleflex Medical (Research Triangle Park, North Carolina), and did not include an inflatable cuff.

The commercial and SL T- and Y-connectors are illustrated in Figure 2. The SL components were constructed to minimize flow disruption by avoiding sudden expansions and contractions in the flow stream and eliminating sharp changes in flow direction. With the SL designs, sigmoid curves are used for changes in flow pathway diameter, and radii of curvatures are applied to smooth changes in flow direction. The final SL designs are the result of an iterative process that implements CFD to minimize aerosol deposition and drug loss. Components are denoted as $\mathrm{CM}$ (commercial) or SL, followed by the geometry type (T- or Y-connector) and the outlet size. For example, CM-Y$8 \mathrm{~mm}$ represents the commercial $\mathrm{Y}$-connector geometry with an outlet for connecting to an 8-mm-internal-diameter ETT.

For the T-connector, an expanded region of aerosol mixing was implemented to accommodate momentum from the aerosol stream and thereby reduce impaction on the lower wall below the nebulizer. SL connections were used to interface the 22-mm ventilator tubing with the expanded mixing region. The overall design of the SL T-connector is similar to the previously proposed $10 \mathrm{~mm}$ tubing design by Longest et $\mathrm{al}^{14}$ for a high-flow oxygen system. The outlet of the T-connector is positioned in the upper portion of the mixing region, which was found to minimize recirculation and deposition.

The SL Y-connector removes a number of sudden expansions and contractions that the aerosol must navigate in the commercial system. A radius of curvature is added to 

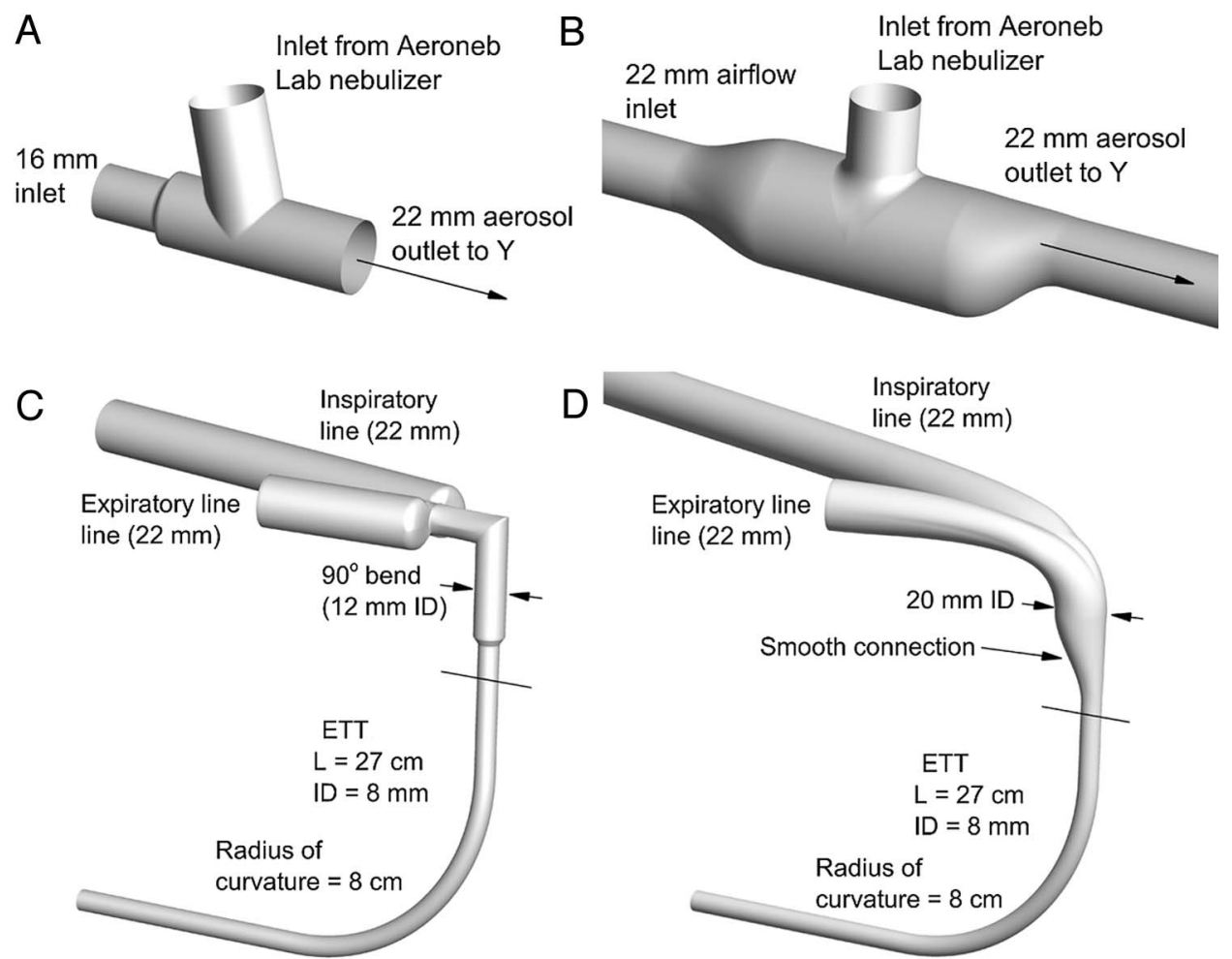

Fig. 2. Commercial and streamlined components for invasive mechanical ventilation. A: Commerical T-connector for 22-mm ventilation tubing. B: Streamlined T-connector for 22-mm ventilation tubing. C: Commercial Y-connector coupled to an endotracheal tube (ETT) with an internal diameter of $8 \mathrm{~mm}$. D: Streamlined Y-connector coupled to an ETT with an internal diameter of $8 \mathrm{~mm}$. L = length; ID $=$ internal diameter.

the $90^{\circ}$ bend region of the Y-connector to minimize flow disruption and aerosol impaction. Identical ETTs are implemented for comparison of the commercial and SL designs. It is noted that the SL Y-connector does not increase the volume of the ventilator circuit. The SL T-connector does increase the volume of the inspiratory line. However, this is not a concern because only air from the ventilator (and not expired gas) is expected to be in the inspiratory tubing.

\section{In Vitro Methods}

Aerosol Size Measurement. In vitro experiments were used to determine the initial size distribution of the aerosol produced by the two nebulizers (ie, Aeroneb Lab and eFlow Rapid). In all experiments, the drug formulation was aqueous-based with $0.2 \%$ weight/volume albuterol sulfate (AS) in deionized water. The Aeroneb Lab and eFlow nebulizers were operated for $30 \mathrm{~s}$ in each experiment resulting in average nominal drug doses of $\sim 379 \mu \mathrm{g}$ (average value range $357-408 \mu \mathrm{g}$ ) and $986 \mu \mathrm{g}$ (average value range 922 $1078 \mu \mathrm{g})$, respectively.

To determine the aerodynamic droplet size distribution for both the Aeroneb Lab and eFlow nebulizers, the de- vices were activated directly into an impactor (Andersen Cascade Impactor $[\mathrm{ACI}]$, Graseby Andersen, Smyrna, Georgia). The nebulizers and ACI were placed in an environmental cabinet (Espec, Hudsonville, Michigan) and maintained at $25^{\circ} \mathrm{C}$ and $93 \%$ relative humidity. A gap distance of $\sim 10 \mathrm{~mm}$ separated the Aeroneb outlet and the ACI inlet, allowing for the entrainment of humidified air at a rate of $28.3 \pm 2 \mathrm{~L} / \mathrm{min}$, while the eFlow was connected directly to the ACI to allow for entrainment of humidified air through the nebulizer. Constant temperature and nearly saturated relative humidity conditions prevented a size change of the aerosol arising from unwanted evaporation and condensation during measurement.

Drug deposition on the ACI collection plates for the initial size experiments was determined following washings using appropriate volumes of deionized water (10$20 \mathrm{~mL}$ ). The solutions were then assayed using a validated high-performance liquid chromatography-ultraviolet assay method for AS. The mass of AS on each impaction plate was determined and used to calculate the aerodynamic particle size distribution of the drug. The mass median aerodynamic diameter (MMAD) was defined as the particle size at the 50th percentile on a cumulative percentage mass undersize distribution (D50) using linear interpola- 
tion. The mass of formulation nebulized was determined by weighing the nebulizer before and after each experiment. The AS solution concentration and the mass of formulation nebulized were used to determine the nominal dose of AS delivered.

Deposition and ED. In vitro experiments were conducted to determine aerosol drug deposition within individual components of the delivery system as well as the ED exiting the ETT at a steady-state flow of $30 \mathrm{~L} / \mathrm{min}$. In the experimental system, a humidification unit (2000i, Vapotherm, Stevensville, Maryland) was implemented to supply steadystate air flow with a relative humidity of $>90 \%$ and a temperature of $25^{\circ} \mathrm{C}$. The in vitro model was tested under ambient room conditions $\left(23-25^{\circ} \mathrm{C}\right)$. The use of $25^{\circ} \mathrm{C}$ air produced a thermally neutral system and prevented condensation of the high humidity air flow. In the experiments, the expiratory side of the Y-connector was closed to allow for only inspiratory air flow. The dose emitted from the ETT was captured on a high-efficiency filter (Pulmoguard II, Quest Medical, Brockton, Massachusetts).

The SL components developed through CFD simulations were constructed using a rapid prototyping process. Specifically, the prototyping process (Viper SLA system, 3D Systems, Valencia, California) was used to construct the SL T-connector and Y-connector designs using Accura 60 clear plastic resin. The resolution of the prototyper was $0.1 \mathrm{~mm}$, and the final surface finish of the SL components was smooth.

Drug deposition in the nebulizers, in individual system components, and on the outlet filter was determined with a validated high-performance liquid chromatography-ultraviolet assay method. As with the particle-sizing experiments, the mass of nebulized formulation was determined by weighing the nebulizers. Drug deposition in the Aeroneb Lab device was below 5\%, whereas deposition in the eFlow device was variable and below $\sim 30 \%$ of the nominal dose. Due to these significant differences in nebulizer deposition and to present a clear picture of deposition in the system components (excluding the nebulizer), drug deposition and ED were calculated as percentages of the mass of drug leaving the nebulizers. At least 4 replications of each experiment were performed. One-way ANOVA, followed by a post hoc two-tailed Student $t$ test was performed using appropriate software (JMP Pro, version 10.0.2, SAS Institute, Cary, North Carolina), with a $P$ value of $<.05$ as the indicator of significance.

\section{CFD Simulations}

Flow Field Dynamics. CFD simulations were conducted to guide the initial development of the SL designs, demonstrate the improved flow characteristics of the SL mod- els, and predict aerosol deposition. Based on the low overall deposition in the T-connectors, described in the results, CFD simulations are reported only for the $\mathrm{Y}$-connector geometries with 8-mm ETTs at the outlets (see Fig. 2C and D) in this study. Isothermal, incompressible, and constant property flow was assumed in all cases. For inlet flows of 30 and $45 \mathrm{~L} / \mathrm{min}$ and an 8-mm ETT, the maximum Reynolds numbers in the tubes are 5,170 and 7,760, respectively, which indicate turbulent flow and the potential for turbulent aerosol dispersion. Turbulence in the components will be amplified in the presence of changes in flow direction and constrictions.

The flow fields in the Y-connector geometries were solved using Fluent 12 (Ansys, Canonsburg, Pennsylvania) with a steady solution and the low Reynolds number $k$ - $\omega$ two-equation turbulence model. This turbulence model was selected based on a combination of its accuracy and high efficiency, compared with more complex methods, such as large eddy simulation. Considering aerosol transport, the low Reynolds number $k-\omega$ model was previously shown to accurately predict particle transport and deposition for both monodisperse and polydisperse aerosol distributions in airway models and delivery devices on a regional and highly localized basis. ${ }^{16-19}$

Computational grids of the air-flow passages were constructed based on previously established best practices..$^{20,21}$ The resulting meshes representing the Y-connectors with ETTs for the commercial and SL designs (see Fig. 2C and D) contained $\sim 505,000$ and 425,000 cells, respectively. Grid convergence of these meshes was established by comparing with meshes containing at least $30 \%$ fewer cells in each case. These comparisons indicated that there were minimal differences $(<5 \%$ relative error) in the maximum velocity and particle deposition values between the grid initially established in this study and the one with $30 \%$ fewer cells. As a result, the finer meshes (more cells) were considered sufficient and were used in all subsequent simulations.

Particle Dynamics. Droplet trajectories and deposition were determined in the commercial and SL Y-connectors using a previously developed Lagrangian tracking algorithm. ${ }^{22,23}$ Factors affecting particle motion included in the transport equations were drag, gravity, and turbulent dispersion. Previously developed near-wall corrections for interpolating velocity and directionally dependent turbulence were included. ${ }^{22,24}$ Due to the use of humidified air flow in the experiments, droplet evaporation was not included in the model, resulting in the simulation of particle trajectories with unchanging diameters. However, the overall MMAD of the polydisperse aerosol changes as the polydisperse particles move through the system and are selectively filtered by deposition. 
A
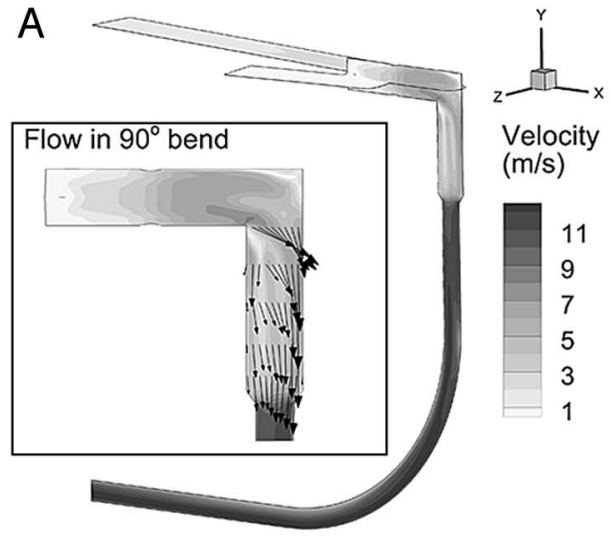
$(\mathrm{m} / \mathrm{s})$

C

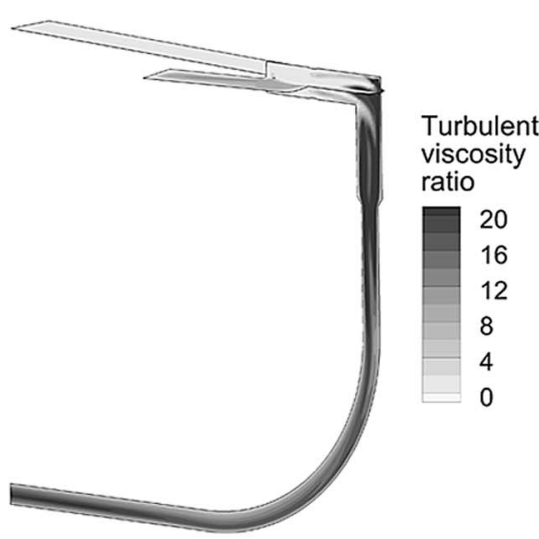

B
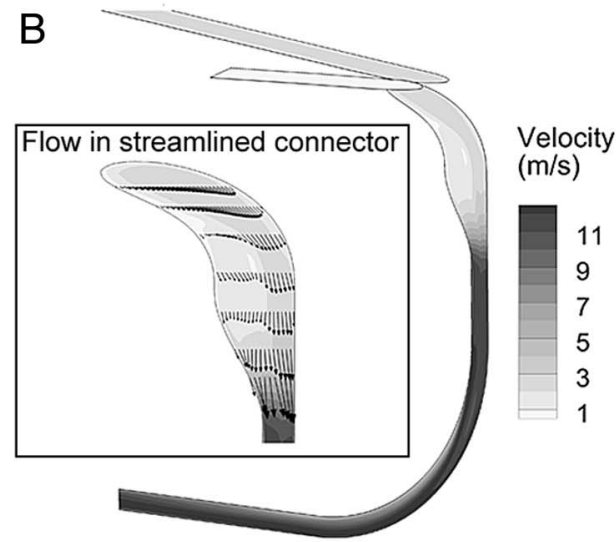

$\mathrm{D}$

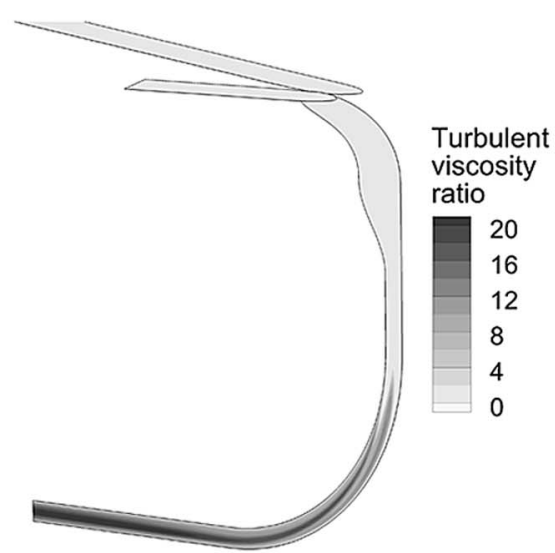

Fig. 3. Contours of flow field properties during steady inspiratory flow at $30 \mathrm{~L} / \mathrm{min}$ including velocity for a commercial $\mathrm{Y}$-connector $(\mathrm{A})$ and a streamlined $Y$-connector (B). Turbulent viscosity ratio for a commercial Y-connector coupled to an 8-mm endotracheal tube (ETT) (C) and a streamlined Y-connector coupled to an 8-mm ETT (D).

CFD simulations considered the polydisperse aerosol distribution determined for the Aeroneb Lab device at flows of 30 and $45 \mathrm{~L} / \mathrm{min}$. To resolve this polydisperse aerosol distribution, 27,000 representative particles were simulated. It was determined that increasing the number of representative particles had a negligible effect on the predicted particle DFs.

To determine an optimal particle size for maximum ED through the SL system, CFD simulations were conducted with monodisperse aerosols ranging from 1 to $4 \mu \mathrm{m}$. In addition, aerosols produced by most mesh nebulizers in a humidified system are known to be bimodal with a significant fraction of particles $>5 \mu \mathrm{m}$, which may limit the performance of the SL components. CFD simulations were used to determine the effects of a hypothetical monomodal log-normal particle size distribution with an MMAD of $3 \mu \mathrm{m}$ and a geometric SD (GSD) of 1.5 in both the CM and SL Y-connector geometries. CFD predictions of DF are based on the initial drug mass of the polydisperse or monodisperse aerosol entering the geometry, unless otherwise noted.

\section{Results}

\section{Flow Field Characteristics}

Velocity fields in the commercial and SL Y-connectors with an 8-mm ETT are compared in Figure 3A and B. In the junction region, where a majority of deposition is expected to occur, velocities are significantly higher for the CM-Y-8 mm model, due to the sharp change in flow direction, compared with the SL design. In contrast, the junction region of the SL-Y-8 mm geometry is observed to provide a larger effective area for flow direction change, which eliminates detachment of the flow profile and reduces velocity values. The reduction in velocity associated with the SL-Y-8 mm geometry is expected to reduce deposition by inertial impaction. The velocity profiles in the ETTs appear similar, as expected.

Figure $3 \mathrm{C}$ and D presents the turbulent viscosity ratio, which is calculated as follows:

$$
\left(\nu+\nu_{\mathrm{T}}\right) / \nu
$$




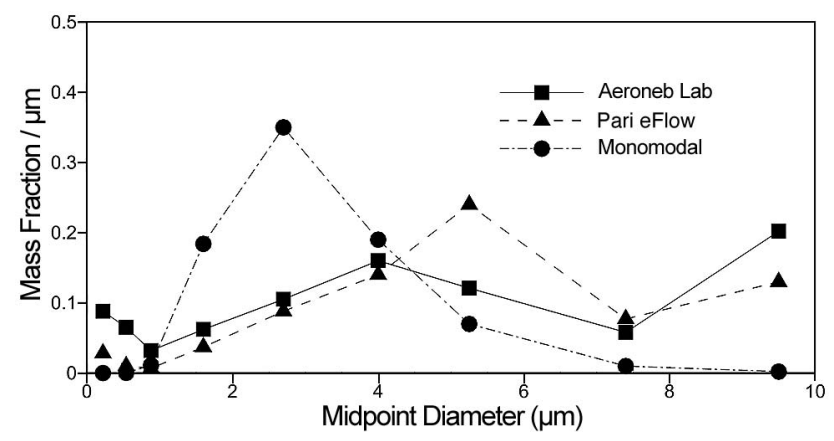

Fig. 4. Polydisperse particle size distributions generated by the Aeroneb Lab and Pari eFlow nebulizers, as well as a hypothetical monomodal log-normal particle distribution.

where $\nu$ and $\nu_{\mathrm{T}}$ are the molecular and turbulent kinematic viscosities, respectively. In turbulence modeling, the effects of turbulence are approximated as an additional viscosity, which acts on the flow field like a molecular viscosity in laminar flow. The turbulent viscosity ratio physically approximates the level that turbulence influences drag and mixing in the flow. Turbulence increases aerosol deposition based on particle dispersion in the multiscale eddies. Figure 3C illustrates the idea that turbulent flow dominates the junction region of the CM-Y-8 mm design and continues into the ETT. In contrast, the viscosity ratio indicates that very little turbulence occurs in the junction region of the SL design, with values $>20$-fold lower than in the CM-Y-8 mm model. In the SL geometry, turbulent flow is initiated just upstream of the ETT bend and continues through the ETT at a level similar to the commercial case.

\section{Initial Particle Size Distribution}

Polydisperse size distributions for the Aeroneb Lab and eFlow Rapid nebulizers are displayed in Figure 4, based on measurements obtained with the ACI. Both nebulizers are observed to produce a bimodal droplet distribution in the presence of humidified air. The larger droplets in these distributions are expected to be deposited in both the CM and SL Y-connector geometries. The MMAD (and GSD) values for the Aeroneb Lab and eFlow Rapid nebulizers were $4.84 \mu \mathrm{m}$ (5.7) and $5.30 \mu \mathrm{m}$ (1.6), respectively. A monomodal particle size distribution with an MMAD of $3.0 \mu \mathrm{m}$ and a GSD of 1.5 was also considered with the CFD model (see Fig. 4). Evaluation of this monomodal distribution allows for evaluation of the SL geometry with a target polydisperse aerosol.

\section{In Vitro Deposition of Drug Mass}

The DF of a drug in the individual system components is presented in Table 1 based on in vitro experiments for the commercial system. These values are presented as a percentage of the dose emitted from the nebulizer at an air flow of $30 \mathrm{~L} / \mathrm{min}$. DFs in the Aeroneb Lab and eFlow Rapid nebulizers were $\sim 3-5 \%$ and $18-28 \%$ of the nominal dose, respectively (not shown in Table 1). Based on these large differences in nebulizer DF, values reported in Table 1 were based on the dose delivered from the nebulizer. Deposition in the Aeroneb Lab T-connector is relatively low $(<10 \%)$ for the applied steady-state conditions (see Table 1). The eFlow Rapid device was connected directly in line with the Y-connector inlet, and a T-connector was not present. High deposition in the commercial Y-connector (33-39\%) and ETT (9-36\%) resulted in overall device deposition of $58-72 \%$. The filter $\mathrm{DF}\left(\mathrm{DF}_{\text {filter }}\right)$ represents the ED at the ETT outlet. The ED was observed to increase with increasing ETT diameter for the commercial devices.

DF results for the SL components at $30 \mathrm{~L} / \mathrm{min}$ based on in vitro experiments are presented in Table 2 . These experimental results are again based on the ED from the nebulizer. The SL T-connector is observed to significantly reduce deposition by a factor of 2-3 times $(P=.019$, $P<.001$, and $P<.001$ for 7-, 8-, and 9-mm ETTs, respectively). However, deposition in the T-connector is low for both the commercial and SL models. The largest improvement with the SL designs occurs for the Y-connector, where deposition is reduced by a factor of $\sim 4-9$ times $(P<.001$ for all SL Y-connectors vs their respective commercial Y-connectors for Aeroneb and eFlow nebulizers). As a result of reducing deposition in the SL Yconnector geometries, a larger aerosol enters the ETT, which increases deposition in this region. The smallest ETT is observed to have the largest increase in depositional losses.

The ED, which is equal to the $\mathrm{DF}_{\text {filter, }}$, is compared between the two systems as an ED ratio, calculated as follows:

$$
\mathrm{ED} \text { ratio }=\text { streamlined } \mathrm{DF}_{\text {filter }} / \text { commercial } \mathrm{DF}_{\text {filter }}
$$

The improvements in ED associated with streamlining range from 1.1 to 1.5 , with higher ED ratio values associated with larger ETTs (see Table 2).

\section{Validation of CFD Predictions}

Comparisons of in vitro results and CFD predictions for the CM-Y-8 mm and SL-Y-8 mm geometries are illustrated in Figure 5 for a flow of $30 \mathrm{~L} / \mathrm{min}$ and the Aeroneb $\mathrm{Lab}$ aerosol. As the T-connector was not considered in the CFD simulations, predictions were corrected by the T-connector DFs reported in Tables 1 and 2. Overall, very good agreement is observed between the predictions and exper- 
Improving Aerosol Drug Delivery During Mechanical Ventilation

Table 1. Commercial Component Deposition Fraction Based on In Vitro Experiments

\begin{tabular}{|c|c|c|c|c|c|}
\hline Components & T-Connector $(\%)$ & Y-Connector $(\%)$ & ETT (\%) & Device (\%) & Filter (\%) \\
\hline \multicolumn{6}{|l|}{ Aeroneb Lab } \\
\hline CM-Y-7 mm & $7.8 \pm 2.3$ & $36.4 \pm 2.3$ & $24.9 \pm 2.5$ & $69.2 \pm 2.7$ & $30.8 \pm 2.7$ \\
\hline CM-Y-8 mm & $7.1 \pm 1.8$ & $39.9 \pm 2.4$ & $16.6 \pm 1.6$ & $63.6 \pm 1.5$ & $36.4 \pm 1.5$ \\
\hline CM-Y-9 mm & $9.1 \pm 1.3$ & $39.0 \pm 2.6$ & $9.4 \pm 2.6$ & $57.6 \pm 4.3$ & $42.4 \pm 4.3$ \\
\hline \multicolumn{6}{|l|}{ eFlow } \\
\hline CM-Y-7 mm & & $37.0 \pm 5.8$ & $35.5 \pm 6.6$ & $72.4 \pm 0.8$ & $27.6 \pm 0.8$ \\
\hline CM-Y-8 mm & & $32.6 \pm 3.2$ & $31.0 \pm 2.3$ & $63.6 \pm 4.9$ & $36.4 \pm 4.9$ \\
\hline CM-Y-9 mm & & $33.9 \pm 4.6$ & $26.7 \pm 9.0$ & $60.6 \pm 4.6$ & $39.4 \pm 4.6$ \\
\hline
\end{tabular}

Data are the mean \pm SD deposition fraction as a percentage of dose emitted from the nebulizer at a flow of $30 \mathrm{~L} / \mathrm{min}$. $\mathrm{ETT}=$ endotracheal tube

$\mathrm{CM}=$ commercial

Table 2. Streamlined Component Deposition Fraction Based on In Vitro Experiments and Emitted Dose Ratio

\begin{tabular}{|c|c|c|c|c|c|c|}
\hline Components & T-Connector $(\%)$ & Y-Connector (\%) & $\operatorname{ETT}(\%)$ & Device (\%) & Filter $(\%)$ & ED Ratio* \\
\hline \multicolumn{7}{|l|}{ Aeroneb Lab } \\
\hline SL-Y-7 mm & $4.8 \pm 0.6$ & $5.6 \pm 0.8$ & $55.9 \pm 1.6$ & $66.3 \pm 2.0$ & $33.7 \pm 2.0$ & 1.1 \\
\hline SL-Y-8 mm & $3.1 \pm 0.6$ & $5.4 \pm 1.3$ & $40.1 \pm 8.5$ & $48.6 \pm 9.9$ & $51.4 \pm 9.9$ & 1.4 \\
\hline SL-Y-9 mm & $2.0 \pm 1.2$ & $4.3 \pm 1.2$ & $31.6 \pm 4.7$ & $37.9 \pm 6.7$ & $62.1 \pm 6.7$ & 1.5 \\
\hline \multicolumn{7}{|l|}{ eFlow } \\
\hline SL-Y-7 mm & & $6.5 \pm 2.1$ & $58.5 \pm 3.4$ & $65.0 \pm 2.5$ & $35.0 \pm 2.5$ & 1.3 \\
\hline SL-Y-8 mm & & $8.6 \pm 1.0$ & $43.9 \pm 4.7$ & $52.4 \pm 5.3$ & $47.6 \pm 5.1$ & 1.3 \\
\hline SL-Y-9 mm & & $5.2 \pm 1.6$ & $37.2 \pm 9.2$ & $42.4 \pm 8.1$ & $57.6 \pm 8.1$ & 1.5 \\
\hline $\begin{array}{l}\text { Data are mean } \pm \text { SD d } \\
{ }^{*} \text { Calculated as (strea } \\
\mathrm{ED}=\text { emitted dose } \\
\mathrm{SL}=\text { streamlined } \\
\mathrm{ETT}=\text { endotracheal } \\
\mathrm{DF}=\text { deposition frac }\end{array}$ & $\begin{array}{l}\text { fraction as a percentage } \\
\left.F_{\text {filter }}\right) /\left(\text { commercial } D_{\text {filte }}\right.\end{array}$ & nitted from the nebulizer a & f $30 \mathrm{~L} / \mathrm{min}$. & & & \\
\hline
\end{tabular}

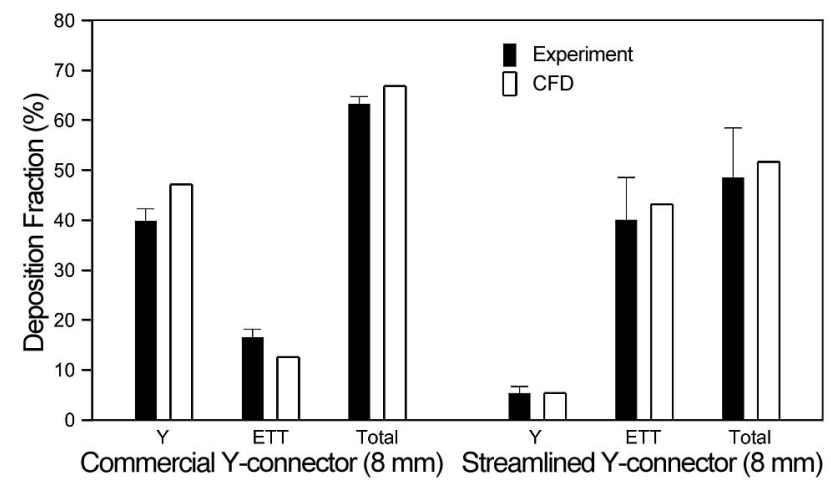

Fig. 5. Comparison of experimental and computational fluid dynamics (CFD) predicted deposition fractions in the invasive delivery system based on dose emitted from the nebulizer at a flow of $30 \mathrm{~L} / \mathrm{min}$ for the Aeroneb Lab aerosol. Error bars indicate \pm one $\mathrm{SD}$ of the experimental data. ETT = endotracheal tube.

iments for DF on an individual component and total drug loss basis. Small overpredictions in the Y-connector and underpredictions in the ETT may be the result of some of the deposited liquid droplets flowing from the Y-connector to the ETT region in the experiments. General agreement between the CFD predictions and experiments instills confidence that the model can accurately predict deposition for both monodisperse and polydisperse aerosols.

\section{CFD Predictions for Monodisperse Aerosols}

DFs for monodisperse aerosols $(1-4 \mu \mathrm{m})$ at flows of 30 and $45 \mathrm{~L} / \mathrm{min}$ are presented in Table 3 for the CM-Y- $8 \mathrm{~mm}$ geometry as well as ED at the outlet based on CFD predictions. Increasing the flow from 30 to $45 \mathrm{~L} / \mathrm{min}$ is observed to largely increase the DFs in both the Y-connector and ETT. Similar results are presented in Table 4 for the SL-Y-8 mm geometry with ED ratios. It is observed that as particle size increases, the ED ratio increases with a maximum value of 1.9 for $4-\mu \mathrm{m}$ particles and a flow of $45 \mathrm{~L} /$ min. The ED ratio is also observed to increase with increasing flow. As a result, the SL components become 
Table 3. Deposition Fraction and Emitted Dose as a Percentage of Mass Entering the Y-Connector for Monodisperse Aerosols in the Commercial System Based on Computational Fluid Dynamic Predictions

\begin{tabular}{lccrc}
\hline $\begin{array}{c}\text { Particle Size } \\
(\mu \mathrm{m})\end{array}$ & $\begin{array}{c}\text { Air-Flow } \\
(\mathrm{L} / \mathrm{min})\end{array}$ & $\begin{array}{c}\text { Y-Connector } \\
(\%)\end{array}$ & $\begin{array}{r}\text { ETT } \\
(\%)\end{array}$ & $\begin{array}{c}\text { ED } \\
(\%)\end{array}$ \\
\hline 1 & 30 & 3.2 & 1.5 & 95.3 \\
& 45 & 4.7 & 1.5 & 93.8 \\
2 & 30 & 9.5 & 4.5 & 86.0 \\
& 45 & 12.8 & 11.2 & 76.0 \\
3 & 30 & 20.1 & 10.9 & 69.0 \\
& 45 & 32.5 & 20.5 & 47.0 \\
4 & 30 & 36.0 & 17.2 & 46.8 \\
& 45 & 51.1 & 25.2 & 23.7
\end{tabular}

$\overline{\text { ETT }=\text { endotracheal tube }}$

$\mathrm{ED}=$ emitted dose

Table 4. Deposition Fraction and Emitted Dose as a Percentage of Mass Entering the Y-Connector for Monodisperse Aerosols in the Streamlined System Based on Computational Fluid Dynamic Predictions

\begin{tabular}{lccccc}
\hline $\begin{array}{c}\text { Particle Size } \\
(\mu \mathrm{m})\end{array}$ & $\begin{array}{c}\text { Air-Flow } \\
(\mathrm{L} / \mathrm{min})\end{array}$ & $\begin{array}{c}\text { Y-Connector } \\
(\%)\end{array}$ & $\begin{array}{c}\text { ETT } \\
(\%)\end{array}$ & $\begin{array}{c}\text { ED } \\
(\%)\end{array}$ & $\begin{array}{c}\text { ED } \\
\text { Ratio* }\end{array}$ \\
\hline 1 & 30 & 1.0 & 0.7 & 98.3 & 1.0 \\
& 45 & 3.2 & 1.1 & 95.7 & 1.0 \\
2 & 30 & 1.6 & 4.5 & 93.9 & 1.1 \\
& 45 & 6.2 & 10.8 & 83.0 & 1.1 \\
3 & 30 & 2.1 & 12.6 & 85.3 & 1.2 \\
& 45 & 7.7 & 27.3 & 65.0 & 1.4 \\
4 & 30 & 3.6 & 26.0 & 70.4 & 1.5 \\
& 45 & 11.9 & 43.1 & 45.0 & 1.9
\end{tabular}

* Calculated as (Streamlined ED)/(Commercial ED).

ETT $=$ endotracheal tube

$\mathrm{ED}=$ emitted dose

more effective at transmitting the aerosol as flow is increased compared to the commercial designs on a relative basis. However, the ED value decreases as flow is increased due to increasing inertial impaction and turbulence.

Figure 6 illustrates local deposition patterns for a $4-\mu \mathrm{m}$ particle and a flow of $30 \mathrm{~L} / \mathrm{min}$ based on CFD predictions. As expected, particles are observed to collect in regions of sudden expansion, contraction, and sharp changes in direction for the commercial geometry. The commercial and SL geometries demonstrate similar deposition in the ETT for this monodisperse aerosol.

\section{CFD Predictions for a Monomodal Aerosol}

For the monomodal aerosol with an MMAD (and GSD) of $3 \mu \mathrm{m}(1.5)$, regional deposition and ED are presented in
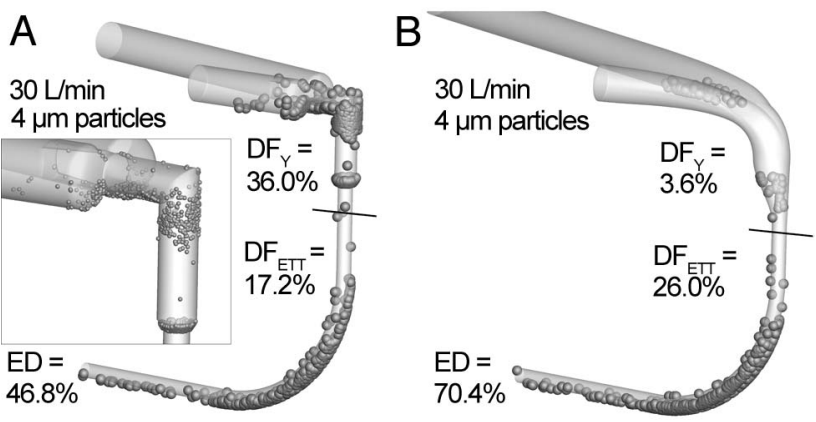

Fig. 6. Predictions of local deposition based on computational fluid dynamics simulations for a flow rate of $30 \mathrm{~L} / \mathrm{min}$ and $4-\mu \mathrm{m}$ monodisperse particles for an 8-mm commercial $\mathrm{Y}$-connector $(\mathrm{A})$ and an 8-mm streamlined $\mathrm{Y}$-connector $(\mathrm{B})$. DF = deposition fraction. $\mathrm{ED}=$ emitted dose.
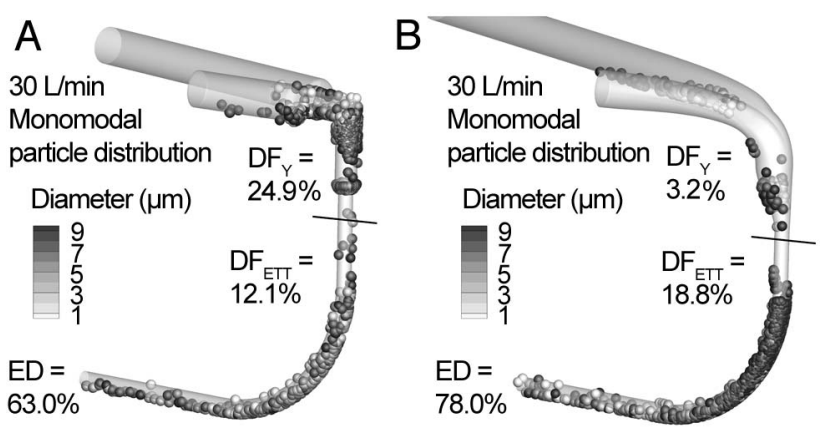

Fig. 7. Predictions of local deposition based on computational fluid dynamics simulations for a flow rate of $30 \mathrm{~L} / \mathrm{min}$ with polydisperse monomodal particle distribution for an 8- $\mathrm{mm}$ commercial Y-connector (A) and an 8-mm streamlined Y-connector (B). $\mathrm{DF}=$ deposition fraction. $\mathrm{ED}=$ emitted dose.

Figure 7 at a flow of $30 \mathrm{~L} / \mathrm{min}$. As before, deposition in the Y-connector geometry is significantly reduced with the SL model. However, deposition in the ETT is relatively similar between the two cases. The resulting ED ratio based on CFD predictions is 1.2, which is consistent with values observed for the two bimodal commercial aerosols. Based on the CFD calculations, the MMADs (and GSDs) exiting the ETT for the commercial and SL systems were $2.7 \mu \mathrm{m}$ (2.2) and $2.8 \mu \mathrm{m}(2.3)$, respectively, at a flow rate of 30 $\mathrm{L} / \mathrm{min}$. As a result, the SL design is observed to only increase the MMAD (and GSD) of the aerosol entering the lungs by a marginal amount.

\section{Discussion}

The primary outcome of the in vitro component of this study was that the SL approach can significantly improve aerosol drug delivery in the case of adult invasive mechanical ventilation. Increases in delivery associated with the new designs depend on both ETT size and the aerosol device selected. The experimental results indicated a de- 
livery enhancement through the system (ED ratio) of 1.3to 1.5 -fold for standard ETT adult sizes of 8 and $9 \mathrm{~mm}$. For the smaller 7-mm ETT, delivery enhancement was dependent on nebulizer device selection. Specifically, an insignificant $(P=.07)$ enhancement was observed with the Aeroneb Lab aerosol and SL components with the 7-mm ETT $($ ED ratio $=1.1)$. However, a significantly larger change $(P=.001)$ was observed for the SL and the eFlow device with the 7 -mm tube $(\mathrm{ED}$ ratio $=1.3)$. Overall delivery of the aerosol was largely dependent on the size and polydispersity of the droplet distribution and could not be predicted based on MMAD alone. For example, the eFlow device produced an aerosol with a larger MMAD and smaller GSD than the Aeroneb Lab; however, there was no significant difference in the ED (or filter deposition) for the Aeroneb Lab device compared to eFlow for similar ETT size. Based on the experiments and steadystate flow conditions, the highest ED was observed for the eFlow nebulizer, SL components, and a 9-mm ETT (DF filter $=62.7 \%$ ), which also displayed the greatest improvement compared with the commercial design $(\mathrm{ED}$ ratio $=1.5)$.

Primary outcomes from the CFD simulations included good agreement with in vitro deposition and ED results, as well as evaluating the effects of flow, aerosol size, and aerosol size distribution on the performance of the SL components. As described in the results, good agreement was observed between the CFD predictions and in vitro data. Some differences in model and experimental results may be expected from the motion of deposited liquid droplets along the surface of the geometry, especially in the high shear environment of the commercial Y-connector. In the study by Longest et al, ${ }^{14}$ a monodisperse droplet approximation was implemented to predict the performance of SL components in a noninvasive ventilation setup. Improvements in CFD model performance compared with the in vitro results observed in the study by Longest et al ${ }^{14}$ indicate that accounting for the polydisperse aerosol size distribution, especially for a bimodal distribution, is important. As expected, device deposition significantly increased with both particle size and flow. The very low device depositions and high EDs observed for 1- and 2- $\mu \mathrm{m}$ monodisperse aerosols (see Tables 3 and 4) are not practical because of the limitations of current nebulizer technology, and these particle sizes will be largely exhaled and not deposited in the lungs of adults during mechanical ventilation. Interestingly, the CFD results indicate that the relative delivery enhancement associated with the SL components was also increased with increasing particle size and flow. The greatest relative delivery enhancement observed with the SL components was for the maximum monodisperse particle size $(4 \mu \mathrm{m})$ and flow $(45 \mathrm{~L} / \mathrm{min})$ considered, with an ED ratio $=1.9$. This observation implies that the SL components will continue to be effective compared with commercial devices for a range of particle sizes, which typically extends beyond $4 \mu \mathrm{m}$ for commercial products, and for values of inspiratory flow that are higher than the average values considered in this study.

Considering CFD predictions with the monomodal aerosol distribution, depositions in both the Y-connector and ETT for the commercial and SL designs were reduced (see Fig. 7) compared with the bimodal distributions generated using the commercial nebulizers (see Tables 1 and 2). As a result, there may be some advantage to improving the nebulizer device aerosol output so that it can provide a monomodal distribution and an MMAD of $\sim 3.0 \mu \mathrm{m}$ for aerosol delivery during invasive mechanical ventilation. In this scenario, delivery even with commercial components was within the range of values observed for SL components $(E D$ ratio $=1.24)$. Due to the high steady-state ED of the commercial case with the monomodal aerosol, the predicted ED ratio is on the lower end of the spectrum of values for the 8-mm ETT, which ranged from 1.3 to 1.5 in the experiments.

From a clinical perspective, the commercial devices selected are very similar to those used in practice, and the new SL components provide several practical advantages. While not currently in clinical use for ventilation circuits, the two mesh nebulizers selected produce aerosols with sizes (MMAD $=4.84-5.30 \mu \mathrm{m})$ very similar to typically used vibrating mesh devices ${ }^{3,4,25}$ like the AeroNeb Pro (Aerogen Limited), with a MMAD of $4.7 \mu \mathrm{m} .{ }^{26}$ The Aeroneb Lab device was expected to produce aerosol sizes smaller than the commercial Pro version to maximize lung delivery, ${ }^{26}$ but a difference in aerosol size was not observed in this study. Similarly, the output rate of drug mass was similar to clinical devices with $\sim 380 \mu \mathrm{g}$ of drug emitted from the Aeroneb Lab nebulizer in $30 \mathrm{~s}$. Typical doses of AS for ventilated patients are $400 \mu \mathrm{g}$ from metered-dose inhalers ${ }^{2}$ and on the order of $1 \mathrm{mg}$ from nebulizers, ${ }^{4}$ with nebulizer delivery periods of up to $15 \mathrm{~min}$. Based on the use of these clinically relevant devices, the SL components provide a potential reduction in both aerosol delivery time and medication cost. The model drug selected in this study (AS) provides an easily quantifiable marker that is frequently administered as a bronchodilator during mechanical ventilation, but it is both inexpensive and administered in relatively low doses. In comparison, the advantages of the SL designs are better realized when considering medications with long delivery times and high costs. For example, inhaled antibiotics, such as tobramycin, have doses on the order of 10-500 mg. ${ }^{27,28}$ With these pharmaceuticals, reducing the delivery time by a factor of $1.3-1.5$ can be advantageous. Similarly, specialized medications such as inhaled iloprost for pulmonary artery hypertension ${ }^{29}$ typically have very high costs and require multiple administrations per day (6-9 times) where a cost 
savings of $\sim 30-50 \%$ would be substantial. While these savings are not as large as can be achieved with more advanced techniques for efficient respiratory drug delivery, like controlled condensational growth, ${ }^{30-32}$ it should be realized that they are easily achieved by simply replacing the ventilator circuit connectors with new redesigned SL components.

It is not surprising that deposition increased in the ETT with the use of SL components. However, this increase was less than the effective improvements gained in the $\mathrm{T}$-connector and Y-connector, resulting in a consistent improvement in ED with the SL system. In the current study, the ETT size was considered to be a fixed parameter that was selected based on the anatomy of individual patients. ED results across a range of ETT sizes provides valuable information regarding the effect of tube size selection on lung aerosol dose for both conventional and the new SL delivery systems with mesh nebulizers. In comparison with the new SL approach, intracorporeal nebulization catheters provide an alternative approach for aerosol delivery during intubation that avoids aerosol loss in the ventilation circuit. ${ }^{33,34}$ However, it should be realized that these devices may produce relatively large aerosol droplets and have high deposition in the region of spray formation. For example, Selting et $\mathrm{al}^{34}$ report a spray with an MMAD of $>10 \mu \mathrm{m}$ and $\sim 50 \%$ tracheal deposition in a rat lung model. While these devices can provide nearly full lung delivery of the aerosol, they may not be ideal for treating the entire lung or for targeting lower airway delivery.

An important issue in ventilation gas delivery is the rebreathing of expired gas. In a 2-limb ventilator circuit, there is an overlap region starting in the Y-connector and continuing through the ETT that receives both inspired and expired gas. The portion of expired $\mathrm{CO}_{2}$-rich air that is in the overlap region at the end of exhalation is subsequently rebreathed during the next inspiratory phase. Increasing the volume of this overlap region will increase the volume of rebreathed $\mathrm{CO}_{2}$-rich air potentially decreasing the ventilation efficiency. Therefore, it is important that a new Y-connector design does not increase the overlap region. Comparing estimates of overlap regions within the Y-connector geometries, values for the commercial and SL devices were observed to be similar and $\sim 16 \mathrm{~cm}^{3}$, not including the ETT. As a result, the proposed SL design is not expected to increase the amount of $\mathrm{CO}_{2}$-rich air that is rebreathed. In contrast, some current nebulizers recommend positioning the device between the Y-connector and ETT to reduce deposition in the $\mathrm{Y}$ and improve delivery efficiency. These devices include a T-connector that will increase the volume of the overlap region. It is not clear whether prolonged use of this T-connector placement will reduce ventilator efficiency due to increased rebreathing of expired gas. A more comprehensive analysis of this topic is needed, including a CFD estimate of flow in the overlap region for both the commercial and SL Y-connector designs.

A primary limitation of this study is the use of approximate steady-state flow compared with more realistic cyclic flow. It is expected that cyclic flow will significantly reduce the total delivery efficiency observed for both the commercial and SL systems. Sources of enhanced deposition during cyclic flow include impaction of the aerosol from the nebulizer onto the T-connector walls in the period of exhalation flow, increased inertia and turbulence associated with flow acceleration and deceleration, and increased time for gravitational settling. The portion of the aerosol left in the overlap region at the end of inspiration is also lost during exhalation. Furthermore, delivery efficiency is typically overestimated in all in vitro experiments because the exhaled aerosol fraction is neglected. The estimates in this study are intended to represent the relative improvement that can be achieved with SL designs for different characteristic aerosol distributions, droplet sizes, and flows. The steady-state estimate ED values are clearly higher than with cyclic flow. However, it is expected that the relative improvement provided by the SL components and reported by the ED ratio are a reasonable estimate for cyclic flow conditions. With cyclic flow, deposition in the T-connector is expected to increase due to deposition during the period of exhalation. With the use of the SL geometry and cyclic flow, deposition in this region may be decreased based on an expanded volume that is better able to accommodate the incoming aerosol. The SL design also allows for entrainment when the inspiratory phase begins.

Additional limitations of this study include operation at room temperature conditions, one configuration of the Yconnector and ETT tubing, and the use of non-clinically approved nebulizers. Humid air flow was implemented in the current study, which is known to reduce delivery efficiency in ventilation circuits by $\sim 40 \%{ }^{3}$ However, the Y-connector and ETT were at room temperature. To maintain thermodynamic equilibrium, $25^{\circ} \mathrm{C}$ humid air was used. In practice, the ventilator tubing is typically maintained at $37^{\circ} \mathrm{C}$, either through air flow or a heated wire, and the ETT is at body temperature. Additional configurations of the Y-connector may have the inspiratory and expiratory lines turned at $90^{\circ}$ or even $180^{\circ}$ to the ETT. Additional simulations with the inspiratory line and ETT both flowing in the same direction $\left(180^{\circ}\right.$ turn of the ETT tube compared with Fig. 1) for the SL components resulted in no change in performance. Finally, the mesh nebulizers were selected to produce two different polydisperse size distributions along with a hypothetical monomodal distribution. While these size distributions are not available in an approved product for use with mechanical ventilation in humans, 


\section{Improving Aerosol Drug Delivery During Mechanical Ventilation}

they are consistent with typical mesh nebulizers and intended to provide a range of expected performance for in vitro testing and CFD simulations.

In conclusion, a streamlining approach applied to components of invasive mechanical ventilation in adults was observed to reduce device deposition and improve ED. The effectiveness of the SL components depended on both the aerosol device and the size of the ETT selected. For a range of potential polydisperse size distributions and ETTs, a 1.1- to 1.5-fold increase in ED was observed. The effect of ETT size on aerosol ED was considered for commercial as well as SL designs in a mechanical ventilation system. CFD results highlighted reduced flow disruption and turbulence in the junction region of the SL Y, which are expected to be responsible for reduced droplet depositional loss in the system. The relative effectiveness of the SL designs compared with the commercial devices improved for both increasing particle size and increasing flow. Future studies are needed to better evaluate the effect of the SL components in gas delivery, the effect of cyclic flow, and an expanded aerosol generation device base, and to apply the streamlining concept to in vivo ventilation circuits.

\section{REFERENCES}

1. Dhand R. Basic techniques for aerosol delivery during mechanical ventilation. Respir Care 2004;49(6):611-622.

2. Dhand R. Inhalation therapy in invasive and noninvasive mechanical ventilation. Curr Opin Crit Care 2007;13(1):27-38.

3. Dhand R. Aerosol delivery during mechanical ventilation: From basic techniques to new devices. J Aerosol Med Pulm Drug Deliv 2008;21(1):45-60.

4. Ari A, Fink JB. Inhalation therapy in patients receiving mechanical ventilation: an update. J Aerosol Med Pulm Drug Deliv 2012;25(6): 319-332.

5. Fuller HD, Dolovich MB, Posmituck G, Pack WW, Newhouse MT. Pressurized aerosol versus jet aerosol delivery to mechanically ventilated patients: comparison of dose to the lungs. Am Rev Respir Dis 1990;141(2):440-444.

6. Fuller HD, Dolovich MB, Turpie FH, Newhouse MT. Efficiency of bronchodilator aerosol delivery to the lungs from the metered-dose inhaler in mechanically ventilated patients: a study comparing 4 different actuator devices. Chest 1994;105(1):214-218.

7. MacIntyre NR, Silver RM, Miller CW, Schuler F, Coleman RE. Aerosol delivery in intubated, mechanically ventilated patients. Crit Care Med 1985;13(2):81-84.

8. Fink JB, Dhand R, Duarte AG, Jenne JW, Tobin MJ. Aerosol delivery from a metered-dose inhaler during mechanical ventilation: an in vitro model. Am J Respir Crit Care Med 1996;154(2):382-387.

9. Fink JB, Dhand R, Grychowski J, Fahey PJ, Tobin MJ. Reconciling in vitro and in vivo measurements of aerosol delivery from a metered-dose inhaler during mechanical ventilation and defining efficiency-enhancing factors. Am J Respir Crit Care Med 1999;159(1): 63-68.

10. Dhand R. Aerosol therapy in patients receiving noninvasive positive pressure ventilation. J Aerosol Med Pulm Drug Deliv 2012;25(2): 63-78.
11. Ari A, Atalay OT, Harwood R, Sheard MM, Aljamhan EA, Fink JB. Influence of nebulizer type, position, and bias flow on aerosol drug delivery in simulated pediatric and adult lung models during mechanical ventilation. Respir Care 2010;55(7):845-851.

12. Dhand R. Nebulizers that use a vibrating mesh or plate with multiple apertures to generate aerosol. Respir Care 2002;47(12):1406-1416.

13. Ivri E, Fink J. Aerosol delivery apparatus and method for pressureassisted breathing systems. US patent 7,290,541. November 6, 2007.

14. Longest PW, Golshahi L, Hindle M. Improving pharmaceutical aerosol delivery during noninvasive ventilation: effects of streamlined components. Ann Biomed Eng 2013;41(6):1217-1232.

15. Delvadia R, Longest PW, Byron PR. In vitro tests for aerosol deposition. I. Scaling a physical model of the upper airways to predict drug deposition variation in normal humans. J Aerosol Med 2012; 25(1):32-40.

16. Longest PW, Hindle M. Evaluation of the Respimat Soft Mist inhaler using a concurrent CFD and in vitro approach. J Aerosol Med Pulm Drug Deliv 2009;22(2):99-112.

17. Xi J, Longest PW, Martonen TB. Effects of the laryngeal jet on nano- and microparticle transport and deposition in an approximate model of the upper tracheobronchial airways. J Appl Physiol 2008; 104(6): 1761-1777.

18. Longest PW, Vinchurkar S. Validating CFD predictions of respiratory aerosol deposition: effects of upstream transition and turbulence. J Biomech 2007;40(2):305-316.

19. Longest PW, Hindle M, Das Choudhuri S, Xi J. Comparison of ambient and spray aerosol deposition in a standard induction port and more realistic mouth-throat geometry. J Aerosol Sci 2008;39(7): $572-591$.

20. Vinchurkar S, Longest PW. Evaluation of hexahedral, prismatic and hybrid mesh styles for simulating respiratory aerosol dynamics. Comput Fluids 2008;37(3):317-331.

21. Longest PW, Vinchurkar S. Effects of mesh style and grid convergence on particle deposition in bifurcating airway models with comparisons to experimental data. Med Eng Phys 2007;29(3):350-366.

22. Longest PW, Hindle M, Das Choudhuri S, Byron PR. Numerical simulations of capillary aerosol generation: CFD model development and comparisons with experimental data. Aerosol Sci Technol 2007; 41(10):952-973.

23. Longest PW, Kleinstreuer C, Buchanan JR. Efficient computation of micro-particle dynamics including wall effects. Comput Fluids 2004; 33(4):577-601.

24. Longest PW, Xi J. Effectiveness of direct Lagrangian tracking models for simulating nanoparticle deposition in the upper airways. Aerosol Sci Technol 2007;41(4):380-397.

25. Dhand R. New frontiers in aerosol delivery during mechanical ventilation. Respir Care 2004;49(6):666-677.

26. Longest PW, Spence BM, Holbrook LT, Mossi KM, Son Y-J, Hindle M. Production of inhalable submicrometer aerosols from conventional mesh nebulizers for improved respiratory drug delivery. J Aerosol Sci 2012;51:66-80.

27. Geller DE. Aerosol antibiotics in cystic fibrosis. Respir Care 2004; 54(5):658-670.

28. Geller DE, Weers J, Heuerding S. Development of an inhaled drypowder formulation of Tobramycin using PulmoSphereTM technology. J Aerosol Med Pulm Drug Deliv 2011;24(4):175-182.

29. Olschewski H, Hoeper MM, Behr J, Ewert R, Meyer A, Borst MM, et al. Long-term therapy with inhaled iloprost in patients with pulmonary hypertension. Respir Med 2010;104:731-740.

30. Longest PW, Hindle M. Condensational growth of combination drugexcipient submicrometer particles: comparison of CFD predictions with experimental results. Pharm Res 2012;29(3):707-721. 


\section{Improving Aerosol Drug Delivery During Mechanical Ventilation}

31. Hindle M, Longest PW. Evaluation of enhanced condensational growth (ECG) for controlled respiratory drug delivery in a mouththroat and upper tracheobronchial model. Pharm Res 2010;27(9): 1800-1811.

32. Tian G, Longest PW, Su G, Hindle M. Characterization of respiratory drug delivery with enhanced condensational growth (ECG) using an individual path model of the entire tracheobronchial airways. Ann Biomed Eng 2011;39(3):1136-1153.
33. Tronde A, Baran G, Eirefelt S, Lennernas H, Bengtsson UH. Miniaturized nebulization catheters: a new approach for delivery of defined aerosol doses to the rat lung. J Aerosol Med 2002;15(3):283296.

34. Selting K, Essman S, Reinero C, Branson KR, Henry CJ, Owen N, et al. Targeted combined aerosol chemotherapy in dogs and radiologic toxicity grading. J Aerosol Med Pulm Drug Deliv 2011;24(1): 43-48. 